\title{
Free boundary problem for one-dimensional compressible Navier-Stokes equations with temperature dependent viscosity and heat conductivity
}

Tuowei CHEN ${ }^{1}$ and yongqian zhang ${ }^{1}$

${ }^{1}$ Fudan University

May 5, 2020

\begin{abstract}
We prove the existence and uniqueness of global strong solution to the free boundary problem in one dimensional compressible Navier-Stokes system for the viscous and heat conducting ideal polytropic gas flow, when the viscosity and heat conductivity depend on temperature in power law of Chapman-Enskog and the data is in the neighborhood of some background solution at initial time. We also study the large time behavior of the solution and obtain its decay property.
\end{abstract}

\section{Hosted file}

1DNS-CHZ200120.pdf available at https://authorea.com/users/300167/articles/429857free-boundary-problem-for-one-dimensional-compressible-navier-stokes-equations-withtemperature-dependent-viscosity-and-heat-conductivity 